\title{
Preparation of a Au SERS substrate and its application in the in-situ Raman spectroelectrochemistry study of $\mathrm{Li}_{2} \mathrm{CO}_{3}-\mathrm{K}_{2} \mathrm{CO}_{3}$ molten salt
}

\author{
Jiangyu Yu ${ }^{l}$, Chengyuan Liu ${ }^{1}$, Xianwei Hu ${ }^{1, *}$, Tian Yuan ${ }^{l}$, Yifan Zhang ${ }^{1}$, Zhaowen Wang ${ }^{1}$, Wuren Ji ${ }^{2, *}$ \\ ${ }^{1}$ School of Metallurgy, Northeastern University, Shenyang 110819, China \\ ${ }^{2}$ Northwest Research Institute of Mining and Metallurgy, Baiyin 730900, China \\ *E-mail: huxw@smm.neu.edu.cn, tomcat3164@163.com
}

Received: 30 July 2021 / Accepted: 8 September 2021 / Published: 10 October 2021

\begin{abstract}
Molten carbonate is often used as a medium for the decomposition of carbon dioxide to produce carbon and oxygen. In-situ Raman spectroelectrochemistry is an effective means to study species changes and the electrochemical reaction mechanism at the electrode-electrolyte interface. In this paper, a Au surfaceenhanced Raman spectroscopy (SERS) substrate was prepared by an electrochemical oxidationreduction cycle (ORC) process, and the reaction mechanism and intermediate species on this kind of $\mathrm{Au}$ SERS electrode in a $\mathrm{Li}_{2} \mathrm{CO}_{3}-\mathrm{K}_{2} \mathrm{CO}_{3}$ molten salt were studied. The prepared Au SERS substrate has a SERS factor of $1.37 \times 10^{4}$, and it presents excellent uniformity and long-term stability. Although the SERS effect of the Au substrate gradually weakens as the temperature increases, it maintains an acceptable SERS factor of $8.76 \times 10^{2}$ after a high-temperature treatment at $873 \mathrm{~K}$. The cyclic voltammogram result shows that carbon reduction preferentially occurs on an Au electrode during the cathodic electrochemical process in $\mathrm{Li}_{2} \mathrm{CO}_{3}-\mathrm{K}_{2} \mathrm{CO}_{3}$ molten salt. In-situ Raman spectroscopy confirms the existence of the intermediate peroxide ion $\left(\mathrm{O}_{2}{ }^{2-}\right)$ during the electrochemical reduction process.
\end{abstract}

Keywords: In-situ Raman spectroscopy; SERS; Carbonate molten salt; Peroxide ion

\section{$\underline{\text { FULL TEXT }}$}

(C) 2021 The Authors. Published by ESG (www.electrochemsci.org). This article is an open access article distributed under the terms and conditions of the Creative Commons Attribution license (http://creativecommons.org/licenses/by/4.0/). 